\title{
ON ERGODIC SEQUENCES OF MEASURES
}

\author{
J. R. BLUM ${ }^{1}$ AND R. COGBURN
}

ABSTRACT. Let $Z$ be the group of integers and $\bar{Z}$ its Bohr compactification. A sequence of probability measures $\left\{\mu_{n}, n=1,2, \ldots\right\}$ defined on $Z$ is said to be ergodic provided $\mu_{n}$ converges weakly to $\bar{\mu}$, the Haar measure on $\bar{Z}$. Let $A_{n} \subset Z, n=1,2, \ldots$, and define $\mu_{n}$ by $\mu_{n}(B)=$ $\left|A_{n} \cap B\right| /\left|A_{n}\right|$ where $|B|$ is the cardinality of $B$. Then it is easy to show that if $\left|A_{n} \cap A_{n}+k\right| /\left|A_{n}\right| \rightarrow 1$ for every $k \in Z$, then $\mu_{n}$ is ergodic. Let $0 \leq p_{k} \leq 1$. In this paper we construct (random) sequences $\left\{\mu_{n}\right\}$ which are ergodic, and such that $\lim \left(\left|A_{n} \cap A_{n}+k\right| /\left|A_{n}\right|\right)=p_{k}$, for every $k \in Z$.

1. Introduction. Let $G$ be a locally compact abelian (1.c.a.) group. Let $\left\{\mu_{n}, n=1,2, \ldots\right\}$ be a sequence of probability measures defined on the Borel sets of $G$. We shall say that such a sequence is ergodic provided $\mu_{n}$ converges weakly to $\bar{\mu}$, where $\bar{\mu}$ is Haar measure on the Bohr compactification of $G$. The reason for this terminology is that ergodicity of such a sequence is necessary and sufficient for the generalized mean ergodic theorem to hold: let $\left\{U_{g}, g \in G\right\}$ be any strongly continuous unitary representation $G$ on a Hilbert space $H$. We say that the generalized mean ergodic theorem holds with respect to the sequence $\left\{\mu_{n}\right\}$ provided $\lim _{n \rightarrow \infty} \int_{G} U_{g} f \mu_{n}(d g)=P f$ strongly, for every $f \in H$, where $P$ is the projection of $H$ on the space $\left\{f \mid U_{g} f=f, g \in G\right\}$.

As mentioned above and shown in [1], the generalized mean ergodic theorem holds for a sequence $\left\{\mu_{n}\right\}$ if and only if the sequence is ergodic. Let $\left\{A_{n}\right\}$ be a sequence of Borel subsets of $G$, with $\mu\left(A_{n}\right)<\infty$, where $\mu$ is Haar measure on $G$. For $g \in G$ let $A_{n} g$ be $A_{n}$ translated by $g$. Define the probability measures $\mu_{n}$ by

for $B$ a Borel set.

$$
\mu_{n}(B)=\mu\left(A_{n} \cap B\right) / \mu\left(A_{n}\right)
$$

It is easy to show that if $\lim _{n \rightarrow \infty}\left(\mu\left(A_{n} \cap A_{n} g\right) / \mu\left(A_{n}\right)\right)=1$ for every $g \in G$ then $\mu_{n}$ is an ergodic sequence. Such sequences will exist if and

Received by the editors April 25, 1974.

AMS (MOS) subject classifications (1970). Primary 43 A05.

${ }^{1}$ Research supported by NSF Grant GB-25736.

Copyright $\odot$ 1975, American Mathematical Society 
only if $G$ is $\sigma$-compact. (See, e.g., [2].) That the above condition is not necessary was shown in [1].

Now let $G=Z$, the group of integers, and let $\left\{k_{j}, j=1,2, \ldots\right\}$ be a sequence of positive integers. Let $A_{n}=\left\{k_{1}, \ldots, k_{n}\right\}$ and define $\mu_{n}$ as above by $\mu_{n}(B)=\left|A_{n} \cap B\right| /\left|A_{n}\right|$, when $|A|$ is the cardinality of $A$. Clearly each $\mu_{n}$ may be thought of as a measure on $\bar{Z}$, the Bohr compactification of $Z$, and it follows from the Levy continuity theorem that $\mu_{n}$ is ergodic if and only if

$$
\lim _{n \rightarrow \infty} \frac{1}{n} \sum_{j=1}^{n} e^{2 \pi i k_{j} \alpha}=0 \text { for } 0<\alpha<1 .
$$

From the criterion :entioned above it follows that such sequences will have this property provided $\left|A_{n} \cap A_{n}+k\right| /\left|A_{n}\right| \rightarrow 1$ for every integer $k$, where

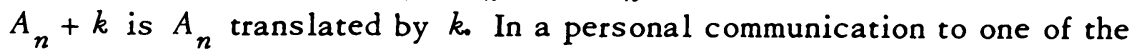
authors, Niederreiter [3] proved that given $p$, with $0 \leq p \leq 1$, and a positive integer $k$, there exists (in fact, he constructed it) a sequence $\left\{k_{j}\right\}$ such that

$$
\lim _{n \rightarrow \infty} \frac{\left|A_{n} \cap A_{n}+k\right|}{\left|A_{n}\right|}=p,
$$

and such that the corresponding measures $\left\{\mu_{n}\right\}$ are ergodic. In this note we prove that given $p_{k}$ with $0 \leq p_{k} \leq 1$, there exist (uncountably many) random sequences $\left\{k_{j}\right\}$ such that

(a) the corresponding sequences of measures are ergodic, and

(b) for every integer $k \neq 0$ we have

$$
\lim _{n \rightarrow \infty} \frac{\left|A_{n} \cap A_{n}+k\right|}{\left|A_{n}\right|}=p_{k} .
$$

In fact we show that (a) and (b) are true on a set of probability one.

As mentioned above, what must be shown is that (1) holds for all $\alpha$. For the kind of sequences we construct it was shown by Robbins [4] that this holds for each $\alpha$ on a set of probability one, and the problem is to construct a single set of probability one such that the limiting relation holds for all a simultaneously. We also consider this problem for the group of reals.

2. The main result. Let $X_{1}, X_{2}, \ldots$ be a sequence of independent, identically distributed random variables with characteristic function

$$
\phi(\alpha)=E e^{i \alpha X_{1}}
$$

Let $S_{n}=X_{1}+\cdots+X_{n}$ and

$$
T_{n}(\alpha)=\frac{1}{n} \sum_{k=1}^{n} e^{i \alpha S_{k}}
$$


Theorem. If $\phi(\alpha) \neq 1$ for $\beta \leq \alpha \leq \gamma$ and if $E\left(X_{1}\right)$ is finite, then $\sup _{\beta \leq a \leq \gamma}\left|T_{n}(\alpha)\right| \rightarrow 0$ almost surely as $n \rightarrow \infty$.

Proof. It is shown in the Lemma below that $\sup _{\beta \leq \alpha \leq \gamma} E\left|T_{n}(\alpha)\right|^{4}=$ $O\left(n^{-2}\right)$. Now let $k$ be integer valued and set

$$
A_{n}=\left[\max _{\beta \leq k / n^{9 / 7} \leq \gamma}\left|T_{n}\left(k / n^{9 / 7}\right)\right| \leq 1 / n^{1 / 7}\right] .
$$

Then, using Boole's and Chebychev's inequalities and the Lemma,

$$
\begin{aligned}
P\left(A_{n}^{C}\right) & \leq \sum_{k: \beta \leq k / n^{9 / 7} \leq \gamma} P\left[\left|T_{n}\left(k / n^{9 / 7}\right)\right|^{2} \geq 1 / n^{2 / 7}\right] \\
& =\sum_{k: \beta \leq k / n^{9 / 7} \leq \gamma} O\left(n^{-10 / 7}\right)=O\left(n^{-1 / 7}\right) .
\end{aligned}
$$

Also

$$
\sup _{\alpha}\left|\frac{d}{d \alpha} T_{n}(\alpha)\right| \leq \frac{1}{n} \sum_{k=1}^{n}\left|S_{k}\right|
$$

and, letting $\mu=E\left|X_{j}\right|$,

$$
E\left|S_{k}\right| \leq \sum_{j=1}^{k} E\left|X_{j}\right|=k \mu
$$

Let

$$
B_{n}=\left[\sup _{\alpha}\left|\frac{d}{d \alpha} T_{n}(\alpha)\right| \leq n^{8 / 7}\right]
$$

By Markov's inequality

$$
P\left(B_{n}^{c}\right) \leq \frac{1}{n^{8 / 7}} E\left(\frac{1}{n} \sum_{k=1}^{n}\left|S_{k}\right|\right) \leq \frac{O\left(n^{2}\right)}{n^{15 / 7}}=O\left(n^{-1 / 7}\right) .
$$

On $A_{n} B_{n}$ we have

$$
\sup _{\beta \leq \alpha \leq \gamma}\left|T_{n}(\alpha)\right| \leq \frac{1}{n^{1 / 7}}+\frac{n^{8 / 7}}{2 n^{9 / 7}}=O\left(n^{-1 / 7}\right),
$$

while $P\left(A_{n} B_{n}\right)^{c}=O\left(n^{-1 / 7}\right)$ from the above estimates.

The proof is completed by using the Borel strong law of large numbers argument: for the subsequence $\left\{n^{8}\right\}$,

$$
\sum_{n} P\left(A_{n}{ }^{B}{ }_{n}{ }^{8}\right)^{c}=\sum O\left(n^{-8 / 7}\right)<\infty
$$

and so $\sup _{\beta \leq \alpha \leq \gamma}\left|T_{n^{8}}(\alpha)\right| \rightarrow 0$ almost surely. 
Now for any $m$, there exists an $n$ such that $n^{8} \leq m<(n+1)^{8}$, and

$$
\begin{aligned}
\left|\left(\left|T_{m}(\alpha)\right|-\left|T_{n}(\alpha)\right|\right)\right| & \leq\left|T_{m}(\alpha)-T_{n}(\alpha)\right| \\
& =\left|\frac{1}{m} \sum_{k=n^{8}+1}^{m} e^{i a S_{k}}-\left(\frac{1}{n^{8}}-\frac{1}{m}\right) \sum_{k=1}^{n^{8}} e^{i a S_{k}}\right| \\
& \leq 2 \frac{m-n^{8}}{m}=O\left(\frac{1}{m^{1 / 8}}\right)
\end{aligned}
$$

uniformly in $\alpha$. The Theorem follows.

In the Lemma below we have occasion to use the relation valid for any complex $a$ and $b$ with $|a| \leq 1$ and $a \neq 1$ :

$$
\left|\sum_{j=1}^{\nu} a^{j} b\right|=\left|\frac{a-a^{\nu+1}}{1-a} b\right| \leq \frac{2|b|}{|1-a|}=\frac{2|b|}{|1-\bar{a}|} .
$$

We also use without further comment the fact that, since the $X_{k}$ 's are independent and identically distributed, for any $k>j$,

$$
E e^{i a\left(S_{k}-S_{j}\right)}=\phi(\alpha)^{k-j}
$$

and the fact that $\phi(-\alpha)=\overline{\phi(\alpha)}$ and $|\phi(\alpha)| \leq 1$.

Lemma. If $\phi(\alpha) \neq 1$ for $\beta \leq \alpha \leq \gamma$ then

$$
\sup _{\beta \leq a \leq \gamma} E\left|T_{n}(\alpha)\right|^{4}=O\left(1 / n^{2}\right) .
$$

Proof. First observe that

$$
\begin{aligned}
E\left|T_{n}(\alpha)\right|^{4} & =E\left(T_{n}(\alpha)^{2} \overline{T_{n}(\alpha)^{2}}\right) \\
& =\frac{1}{n^{4}} \sum_{j, k, l, m=1}^{n} E e^{i a\left(s_{j}+s_{k}-s_{l}-s_{m}\right)} \\
& =\frac{1}{n^{4}}\left(\sum_{1}+\sum_{2}+\sum_{3}+\sum_{4}\right)
\end{aligned}
$$

where

$$
\sum_{\nu}(\alpha)=\sum_{j, k, l, m=1 ;|\{j, k, l, m\}|=\nu}^{n} E e^{i a\left(s_{j}+s_{k}-s_{l}-S_{m}\right)} .
$$

The modulus of each term in the sum for $\Sigma_{\nu}$ is at most one, so $\left|\Sigma_{1}\right|=O(n)$ and $\left|\Sigma_{2}\right|=O\left(n^{2}\right)$.

It is best to break $\Sigma_{3}$ into two sums, $\Sigma_{3}^{\prime}$ and $\Sigma_{3}^{\prime \prime}$, where $\Sigma_{3}^{\prime}$ consists of those terms of $\Sigma_{3}$ which for $j=k$ or $l=m$ and $\Sigma_{3}^{\prime \prime}$ consists of those 
terms of $\Sigma_{3}$ for which $|\{j, k\} \cap\{l, m\}|=1$. Then

$$
\begin{aligned}
& \left|\Sigma_{3}^{\prime}(\alpha)\right|=2\left|\operatorname{Re}\left(\sum_{j \neq k \neq l} E e^{i a\left(2 s_{j}-s_{k}-s_{l}\right)}\right)\right| \\
& =4\left|\operatorname{Re}\left(\sum_{j>k>l}\left\{E e^{i a\left(2 s_{j}-s_{k}-s_{l}\right)}+E e^{i a\left(2 s_{k}-S_{j}-S_{l}\right)}+E e^{i a\left(2 s_{l}-S_{j}-S_{k}\right)}\right\}\right)\right| \\
& \left.\left.=4 \mid \operatorname{Re}\left(\sum_{j>k>l}\left\{\phi(2 \alpha)^{j-k} \phi(\alpha)^{k-l}+\overline{\phi(\alpha)}\right)^{j-k} \phi(a)^{k-l}+\overline{\phi(\alpha)}^{j-k} \overline{\phi(2 \alpha}\right)^{k-l}\right\}\right) \mid \\
& \leq \frac{8}{|1-\phi(\alpha)|}\left(\left|\sum_{j>k} \phi(2 a)^{j-k}\right|+\left|\sum_{j>k} \overline{\phi(\alpha)}^{j-k}\right|+\left|\sum_{k>l} \overline{\phi(2 \alpha)^{k-l}}\right|\right) \\
& =O\left(n^{2}\right) /|1-\phi(\alpha)| \text {. }
\end{aligned}
$$

where the inequality uses $(*)$.

Similarly, using $(*)$ in the last equality,

$$
\begin{aligned}
\left|\sum_{3}^{\prime \prime}(\alpha)\right| & =4\left|\sum_{j \neq k \neq l} E e^{i a\left(s_{j}-s_{k}\right)}\right| \\
& =8(n-2)\left|\operatorname{Re}\left(\sum_{j>k} \phi(\alpha)^{j-k}\right)\right|=O\left(n^{2}\right) /|1-\phi(\alpha)| .
\end{aligned}
$$

To estimate $\Sigma_{4}$ we will first write it in terms of ordered summation indices, $j>k>l>m$. There are then six types of terms according to the position of the two positive signs among $\pm S_{j} \pm S_{k} \pm S_{l} \pm S_{m}$. These can be coalesced into three types of terms by adding conjugates, to give

$$
\begin{aligned}
\left|\sum_{4}(\alpha)\right| & =8 \mid \operatorname{Re}\left(\sum _ { j > k > l > m } \left\{E e^{i \alpha\left(s_{j}+s_{k}-s_{l}-s_{m}\right)}+E e^{i \alpha\left(s_{j}-s_{k}+s_{l}-s_{m}\right)}\right.\right. \\
& \left.\left.+E e^{i \alpha\left(s_{j}-s_{k}-s_{l}+s_{m}\right)}\right\}\right) \mid \\
& =8 \mid \operatorname{Re}\left(\sum _ { j > k > l > m } \left\{\phi(\alpha)^{j-k} \phi(2 \alpha)^{k-l} \phi(\alpha)^{l-m}+\phi(\alpha)^{j-k+l-m}\right.\right. \\
& \leq \frac{16}{\mid(1-\phi(\alpha) \mid}\left\{\left|\sum_{k>l>m} \phi(2 \alpha)^{k-l} \phi(\alpha)^{l-m}\right|+2 n\left|\sum_{l>m} \phi(\alpha)^{l-m}\right|\right\} \\
& =O\left(n^{2}\right) /|1-\phi(\alpha)|^{2} .
\end{aligned}
$$

Here $(*)$ is used twice, at the inequality and the last equality.

Combining these estimates, 


$$
\sup _{\beta \leq \alpha \leq \gamma} E\left|T_{n}(\alpha)\right|^{4}=\frac{\left.\alpha n^{2}\right)}{n^{4}} \sup _{\beta \leq \alpha \leq \gamma} \frac{1}{|1-\phi(\alpha)|^{2}} .
$$

The hypothesis of the Lemma, together with the continuity of $\phi$, imply that the supremum is finite, and the assertion is proved.

Let $\mathcal{L}(d)$, for $d>0$, denote the lattice $\{0, \pm d, \pm 2 d, \ldots\}$. We will say that $X$ is an $\mathcal{L}(d)$ lattice variable if $P[X \in \mathscr{L}(d)]=1$ but there is no $d^{\prime}>d$ such that $P\left[X \in \mathscr{L}\left(d^{\prime}\right)\right]=1$. It is well known that $X$ is an $\mathscr{L}(d)$ lattice variable if and only if $\phi(\alpha) \neq 1$ for $0<\alpha<2 \pi / d$ and $\phi(2 \pi / d)=1$.

Corollary 1. If $X_{1}$ is an $\mathfrak{L}(1)$ lattice variable, then there exists a null set $N$ not depending on a such that, except on $N, T_{n}(\alpha) \rightarrow 0$ as $n \rightarrow \infty$ for every $\alpha \neq 0(\bmod 2 \pi)$.

Proof. In this case the $T_{n}(\alpha)$ as well as $\phi(\alpha)$ are periodic of period $2 \pi$. By the Theorem, for any $k>0$, $\sup _{1 / k \leq \alpha \leq 2 \pi-1 / k}\left|T_{n}(\alpha)\right| \rightarrow 0$ as $n \rightarrow \infty$ except on a null set $N_{k}$.

Evidently we can take $N=\bigcup_{k=1}^{\infty} N_{k}$ to be the set specified in the corollary.

We will say that $X$ is a nonlattice variable if $X$ is not an $\mathscr{L}(d)$ lattice variable for any $d>0$ and if $P[X \neq 0]>0$. This is the case if and only if $\phi(\alpha) \neq 1$ for any $\alpha \neq 0$. Then in the same way as for the first corollary we have the following result.

Corollary 2。 If $X_{1}$ is a nonlattice variable, then there exists a null set $N$ not depending on $\alpha$ such that, except on $N, T_{n}(\alpha) \rightarrow 0$ as $n \rightarrow \infty$ for every $\alpha \neq 0$.

Let

$$
r_{n}(\delta)=\frac{\left|\left\{s_{1}, \ldots, s_{n}\right\} \cap\left\{s_{1}+\delta, \ldots, s_{n}+\delta\right\}\right|}{\left|\left\{s_{1}, \ldots, s_{n}\right\}\right|} .
$$

We will consider only the case that $X_{1}>0$ so $\left|\left\{S_{1}, \ldots, S_{n}\right\}\right|=n$.

Lattice case. Let $X_{1}$ be an $\mathcal{L}(1)$ lattice variable. Then

as $n \rightarrow \infty$.

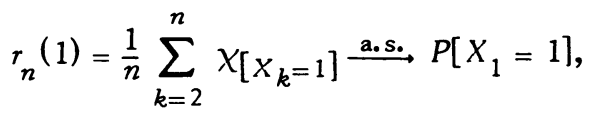

Continuous case. Let $X_{1}$ be a nonlattice variable. Then

$$
\begin{aligned}
r_{n}(\delta) & =\frac{1}{n} \sum_{k=2}^{n} X\left[x_{k}=\delta \text { or } X_{k}+X_{k+1}=\delta \text { or } \cdots \text { or } X_{k}+\cdots+X_{n}=\delta\right] \\
& =\frac{1}{n} \sum_{j=0}^{n-2} \sum_{k=2}^{n-j} \chi_{A_{k, j}}
\end{aligned}
$$


where

$$
A_{k, j}=\left[X_{k}+\cdots+X_{k+j}=\delta\right] \text {. }
$$

Let $A_{k}=\bigcup_{j=0}^{\infty} A_{k, j}$ and let $p_{j}=P\left(A_{1, j}\right)$ and $p=P\left(A_{1}\right)$. Then by the ergodic theorem

$$
\frac{1}{n} \sum_{k=1}^{n} \chi_{A_{k, j}} \stackrel{\text { a.s. }}{\longrightarrow} p_{j}, \quad \frac{1}{n} \sum_{k=1}^{n} \chi_{A_{k}} \stackrel{\text { a.s. }}{\longrightarrow} p
$$

as $n \rightarrow \infty$. But we have $r_{n}(\delta) \leq n^{-1} \sum_{k=2}^{n} \chi_{A_{k}}$ so $\lim \sup r_{n}(\delta) \leq p$. On the other hand, by Fatou's lemma,

$$
\lim \inf r_{n}(\delta) \geq \sum_{j=0}^{\infty} \lim \inf \left(\frac{1}{n} \sum_{k=2}^{n-j} \chi_{A_{k, j}}\right)=\sum_{j=0}^{\infty} p_{j}=p
$$

since $A_{1}=\bigcup_{j} A_{1, j}$ and the sets in the union are disjoint. Thus $r_{n}(\delta) \stackrel{\text { a.s. }}{\longrightarrow} p$, as $n \rightarrow \infty$. Clearly $p=0$ except for at most a countable set of $\delta$ values.

3. Concluding remarks. Now let $X_{1}, X_{2}, \ldots$ be $\mathscr{L}(1)$ random variables. Then it follows from the results in $\$ 2$ that if we define $k_{j}=X_{1}+\cdots+X_{j}$ the corresponding sequences of measures satisfy conditions (a) and (b) of $\$ 1$ with probability one.

The results also apply to the case when $G=R$, the additive group of real numbers. For if $X_{1}, X_{2}, \ldots$ are positive nonlattice random variables we can apply Corollary 2 to show that $\lim _{n} T_{n}(\alpha)=0$ for all $\alpha \neq 0$ with probability one. Thus if $k_{j}=X_{1}+\cdots+X_{j}$, and if $Y(t), t \geq 0$ is a stationary stochastic process we see that the mean ergodic theorem applies to the averages $n^{-1} \sum_{j=1}^{n} Y\left(k_{j}\right)$ provided the process is second order.

\section{REFERENCES}

1. J. R. Blum and B. Eisenberg, Generalized summing sequences and the mean ergodic theorem, Proc. Amer. Math. Soc. 42 (1974), 423-429.

2. E. Hewitt and K. A. Ross, Abstract harmonic analysis. Vol. I: Structure of topological groups. Integration theory, group representations, Die Grundlehren der math. Wissenschaften, Band 115, Academic Press, New York; Springer-Verlag, Berlin, 1963. MR 28 \#158.

3. H. Niederreiter, On a paper of Blum, Eisenberg and Hahn concerning ergodic theory and the distribution of sequences in the Bohr group, Acta. Sci. Math. (to appear).

4. H. Robbins, On the equidistribution of sums of independent random variables, Proc. Amer. Math. Soc. 4 (1953), 786-799. MR 15, 139.

DEPARTMENT OF MATHEMATICS, UNIVERSITY OF NEW MEXICO, ALBUQUERQUE, NEW MEXICO 87106 (Current address of R. Cogburn)

Current address (J. R. Blum): Department of Mathematics, University of Wisconsin-Milwaukee, Milwaukee, Wisconsin 53201 